Supplementary information for:

\title{
Size Effects on Surface Chemistry and Raman Spectra of sub-5 nm Oxidized HPHT and Detonation Nanodiamonds
}

Stepan Stehlik ${ }^{1}$, Michel Mermoux ${ }^{2}$, Bernhard Schummer ${ }^{3}$, Ondrej Vanek ${ }^{4}$, Katerina Kolarova ${ }^{1}$, Pavla Stenclova ${ }^{1}$, Ales Vlk ${ }^{1}$, Martin Ledinsky ${ }^{1}$, Rene Pfeifer ${ }^{1}$, Oleksandr Romanyuk ${ }^{1}$, Ivan Gordeev ${ }^{1}$, Francine Roussel-Dherbey ${ }^{5}$, Zuzana Nemeckova ${ }^{6}$, Jiri Henych ${ }^{6,7}$, Petr Bezdicka ${ }^{6}$, Alexander Kromka ${ }^{1}$, Bohuslav Rezek $^{8}$

${ }^{1}$ Institute of Physics of the Czech Academy of Sciences, Cukrovarnická 10, 16200 Prague 6, Czechia

${ }^{2}$ Univ. Grenoble Alpes, Univ. Savoie Mont Blanc, CNRS, Grenoble INP, LEPMI, 38000 Grenoble, France

${ }^{3}$ Würzburg University, Chair for X-Ray Microscopy, Josef-Martin-Weg 63, 97074 Würzburg, Germany

${ }^{4}$ Department of Biochemistry, Faculty of Science, Charles University, Hlavova 2030/8, 128 40,

Prague, Czechia

${ }^{5}$ Univ. Grenoble Alpes, Grenoble INP, CMTC, 38000 Grenoble, France

${ }^{6}$ Institute of Inorganic Chemistry of the Czech Academy of Sciences, 25068 Husinec-Řež, Czechia

${ }^{7}$ J.E. Purkyně University in Ústí nad Labem, Králova výšina 7,400 96 Ústí nad Labem, Czechia

${ }^{8}$ Faculty of Electrical Engineering, Czech Technical University in Prague, Technická 2, 166 27, Prague,

Czechia

\section{XRD analysis}

We performed the XRD analysis to obtain an average crystallite size in the samples using the Scherrer equation. The diffraction patterns of all the samples along the (111) plane of diamond are shown in Figure S1. It is immediately apparent that the diffraction patterns of the DND samples are very similar to each other with no clear evolution (e.g. broadening) with decreasing size from SD-DND reference to the SR O-DND uc3 fraction. Correspondingly, the XRD-derived crystallite sizes are 2.9-3.0 $\mathrm{nm}$ for all these four samples using cube shape approximation. This is the first indication that the XRD-derived size does not evolve with the decreasing size despite significant narrowing of the particle size distribution (PSD) as well as the shift of the volumetric modes suggested by other techniques. Notably, the diffraction patterns of the SR O-DND uc2 and uc3 fraction contain additional diffraction peak of an unknown impurity, probably $\mathrm{NaCl} 220$ (PDF-4+ \# 00-005-0628). The origin of this contamination is at the moment unknown. In contrast to SR O-DNDs, there is a clear broadening of the 111 diffraction line with the decreasing size in HPHT O-ND samples from $\mathrm{c} 1$ to uc3 fraction and a corresponding decrease of the average crystallite size from $5.1 \mathrm{~nm}$ (c1) across $3.9 \mathrm{~nm}$ (uc2) to $2.6 \mathrm{~nm}$ (uc3) for cube shape approximation. 
(a)

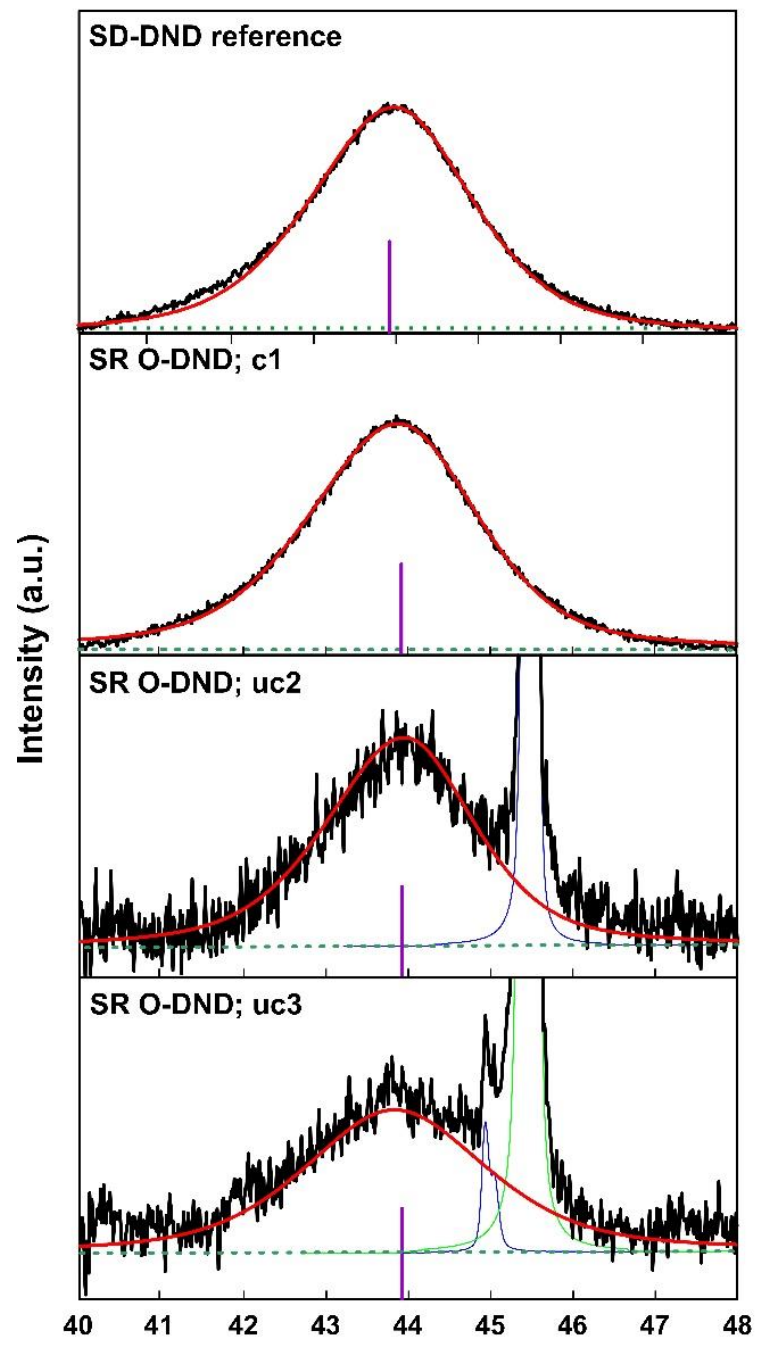

(b)

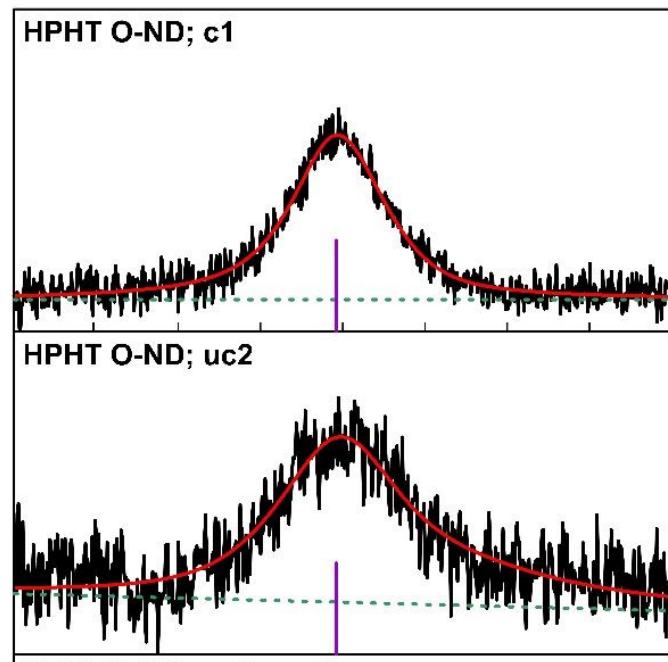

HPHT O-ND; uc3

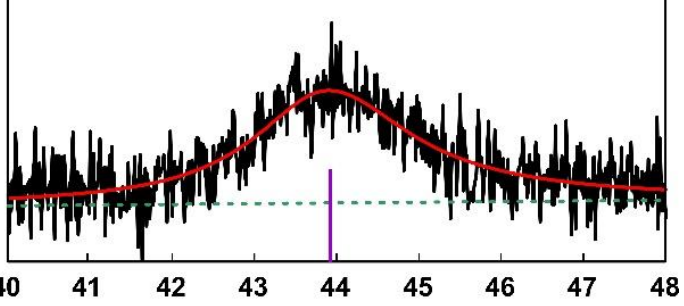

\section{2 theta (deg.)}

Figure S1. Diffraction patterns of the SD-DND-reference, SR O-DND c1, uc2, uc3 fractions (a) and of the HPHT O-ND c1, uc2, uc3 fractions (b). The XRD experimental data are in black, fits of the 111 diffraction line in red and the blue and green correspond to fits of the diffraction line of an unknown impurity (uc2 and $u c 3$ of the SR O-DND samples only). The purple line indicates the position of the 111 diffraction line of diamond according to PDF-4+ \# $00006-0675$ record.

\section{SAXS analysis}

The integral equation of the scattering intensity

$$
I(Q)=(\Delta \rho)^{2} \int|F(Q, r)|^{2} V(r)^{2} N_{p}(r) d r
$$

is used during data analysis with the scattering vector $Q=\frac{4 \pi}{\lambda} \cdot \sin (\theta)$, the scattering angle $\theta$, the scattering contrast $(\Delta \rho)^{2}$ between sample and solvent, the particle form factor $F(Q, r)$, the particles volume $V(r)=\frac{4 \pi}{3} r^{3}$ and radius $r$ as well as the size distribution $N_{p}(r) . N_{P}$ is a histogram with 100 predefined bins between a given diameter range of 5 and $500 \AA$. The height of the bins is then optimized 
to fit the experimental data using e.g. the total non-negative least square method ${ }^{1}$. All particles are assumed to have a constant scattering contrast and the same shape, either using the spherical form factor $^{2}$

$$
\left|F_{S}(Q, r)\right|^{2}=\frac{3 \sin (Q r)-Q r \cos (Q r)}{(Q r)^{3}}
$$

or the rod-like form factor

$$
\left|F_{r}(Q, r)\right|^{2}=A+G_{1} e^{-Q^{2} R g_{1}^{\frac{2}{3}}}+\frac{B_{1}}{\vec{Q}_{*}^{P 1}}
$$

where

$$
\begin{gathered}
G_{2}=1, R g_{2}=\sqrt{r+L^{\frac{2}{12}}}, B_{2}=G_{2} \frac{\pi}{L}, P 2=1, R g_{1}=\frac{\sqrt{3}}{2} r, R g C O_{2}=R g_{1}, G_{1}=\frac{2 G_{2} r}{3 L}, \\
B_{1}=4 G_{2} \frac{L+r}{r^{3} L^{2}}, P_{1}=4, \vec{Q}_{*}=\frac{Q}{\operatorname{erf}\left(Q \frac{R g_{1}}{\sqrt{6}}\right)^{3}}, A=G_{2} e^{Q^{2} R g_{2}^{2 / 3}}+\frac{B_{2}}{\vec{Q}_{*}^{P_{2}}} e^{-R g C O_{2}^{2} Q^{2 / 3}}
\end{gathered}
$$

In addition, it is assumed, that there is no structure factor due to inter-particle interactions. This should be fulfilled, since the samples are highly diluted. The concentrations were: SD DND $=1 \mathrm{mg} / \mathrm{ml}, \mathrm{c1}$ fractions $=\sim 0.5 \mathrm{mg} / \mathrm{ml}$, uc2 fractions $=\sim 0.02 \mathrm{mg} / \mathrm{ml}$, and uc3 fractions $=\sim 0.01 \mathrm{mg} / \mathrm{ml}$ for both SR O DNDs and HPHT O-NDs.

Figure S2 shows the SAXS data of the SD DND reference (a), the three fractions of the HPHT O-NDs (b) and two measureable fractions of the SR O-DNDs (c).

(a)

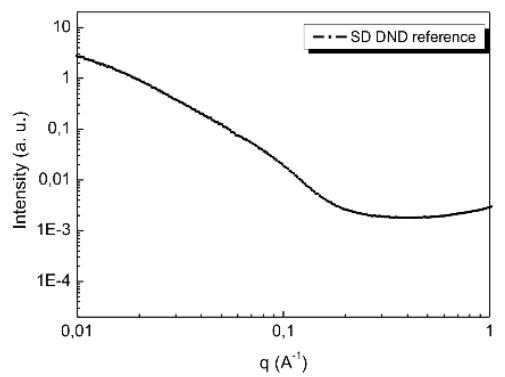

(b)

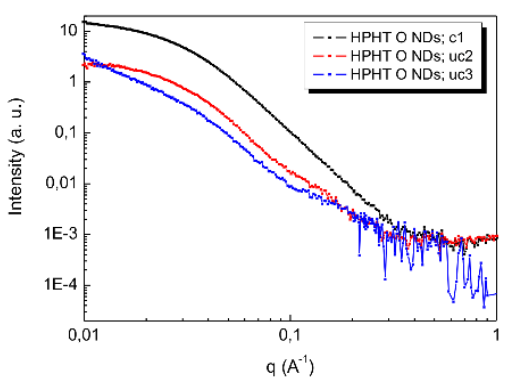

(c)

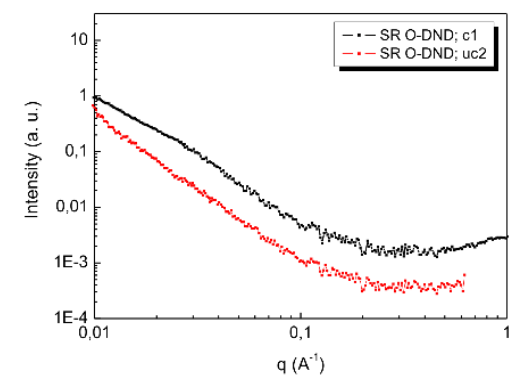

Figure S2. Raw SAXS data of the SD DND reference (a), the three fractions of the HPHT O-NDs (b) and two measureable fractions of the SR O-DNDs (c). 
(a)

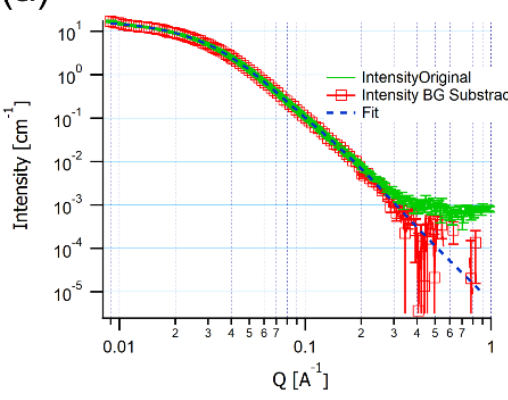

(d)

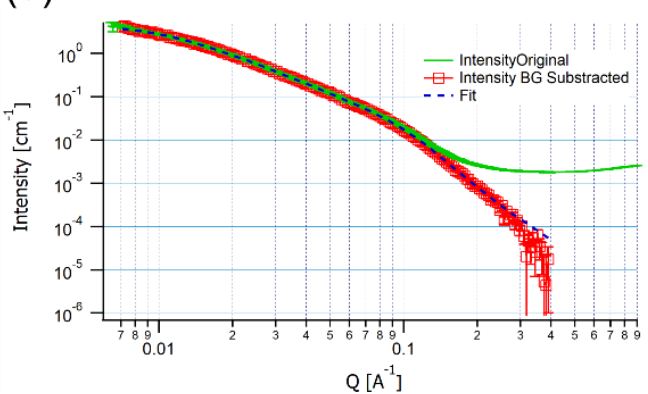

(c)

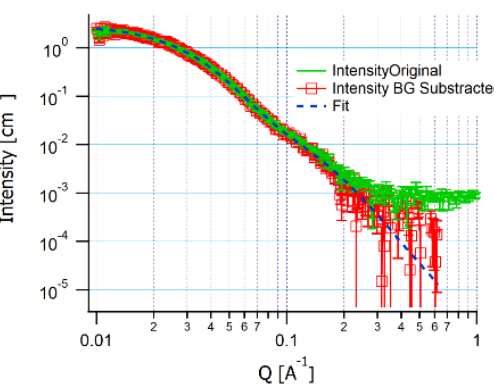

(e)

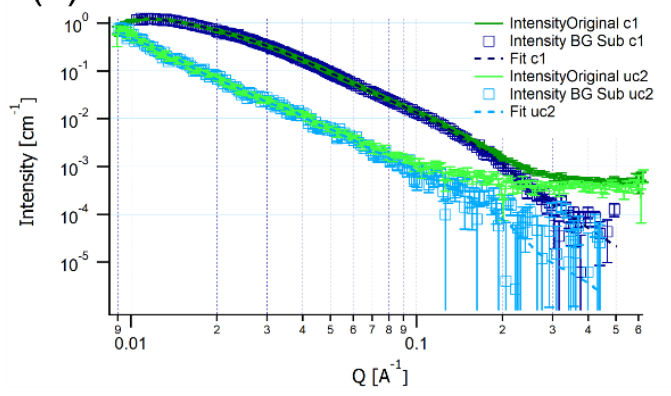

Figure S3. SAXS data, data with subtracted background, and fits of HPHT O-NDs for the c1 (a), uc2 (b) and uc3 (c) fractions, SD DND ref (d) and SR O-DNDs c1 and uc2 (e).

Comparing SAXS curves of the $c 1$, uc2 and uc3 fractions of the HPHT O-NDs in the Figure S3a-c, it is obvious that the intensity in the low $\mathrm{Q}$ area is about one order lower for uc2 and uc 3 fractions because of the decrease of the volume concentration of the particles after the ultracentrifugation. The uc 3 curve does not form a distinct plateau in the low $Q$ area like $c 1$ and uc2, which may signalize agglomeration of the very small particles. In general, all the SAXS data show a smooth decay without any oscillations, which is due to a size distribution of the NDs. The SD DND reference sample (Fig. S3d) shows at low $Q$ values below $0.012 \frac{1}{\AA}$ a $Q^{-1}$ qower law decay, $Q^{-2}$ between 0,2 and $0,7 \frac{1}{\AA}$ and $Q^{-3.5}$ at around $0.11 \frac{1}{\AA}$ with a Guiner knee at around $0.1 \frac{1}{\AA}$. The SR O-DND C1 NDs has similar curve to the SD DND reference but have a $Q^{-0}$ at low q values and the Guiner knee is located $0,11 \frac{1}{\AA}$ meaning slighty smaller aprticles than in the SD DND reference sample. The SR O-DND uc1 sample shows no Guiner knee and a power law decy of $Q^{-2.7}$. The HTPT O-NDs have two Guiner knees at around 0.02 and 0.2 $\frac{1}{\AA}$ separated with a $Q^{-3.6}$ qower law decay. The Guiner knee at around $0.2 \frac{1}{\AA}$ is more expressed for the uc2 and uc3 fractions and corresponds to the sub- $4 \mathrm{~nm}$ ND fraction. Despite the $Q^{-1} / \mathrm{Q}^{-2}$ power law decay, identified in SD DND ref. and SR O-DND c1 (Figure S3d,e), represents rods/disks in theory, we decided to fit all the data (see Fig S3) using a spherical form factor to allow comparability with other measurements (SAXS, DLS, AUC). In addition, a model involving spheres and discs for the SR O-DND c1 sample, for instance, results in nearly the same size distribution (see Fig. S4). 
(a)

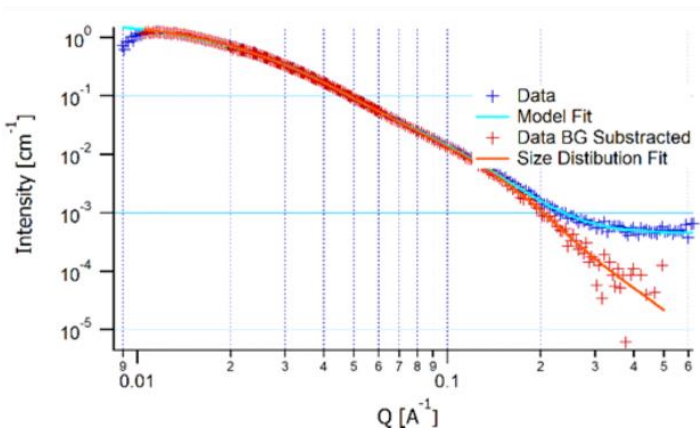

(b)

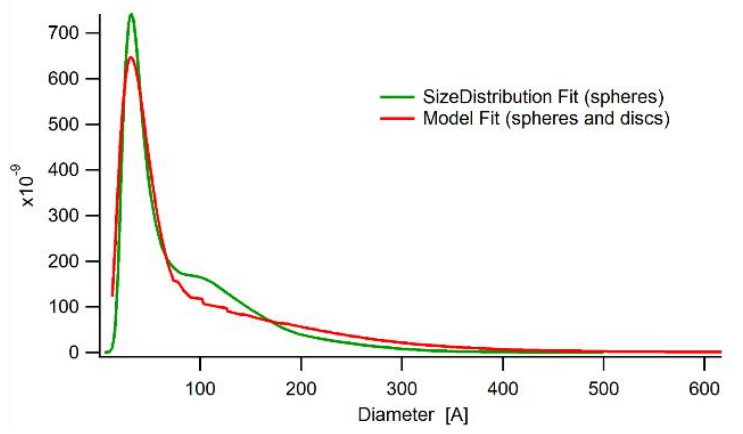

Figure S4. SAXS data and size distribution fit of the SR O-DND c1 using a model involving spheres and discs (a). Comparison of the SD DND ref. size distributions obtained by using model involving spheres and discs (red curve) and spheres only (green curve) (b).

Importantly, the mass/volume fraction of the discs compared to the spheres is nearly 4 times lower which justifies use of the spherical form factor. All size distribution fits (see Figure S3) perfectly match the measured data. Since TEM measurements of HTPT O-NDs show frequent occurrence of elongated, high aspect ratio ND shapes these curves were additionally fitted with rod shape. The length of the rods was fixed at $110 \AA$ since the maximum of the seconds peak's size distribution is located at $110 \AA$.

\section{Raman analysis of the second, more graphitic, HPHT O-ND sample batch}

Figure S5 shows the Raman spectra of three fractions of the second HPHT O-ND sample batch with higher concentration of the $\mathrm{sp}^{2}-\mathrm{C}$ contamination residuals. The Raman spectra were acquired with 785 $\mathrm{nm}$ (a), $514 \mathrm{~nm}$ (b), and $325 \mathrm{~nm}$ (c) excitation. The spectra show that even after relatively harsh oxidation conditions $\left(450^{\circ} \mathrm{C}, 5 \mathrm{~h}\right)$, the $\mathrm{sp}^{2}-\mathrm{C}$ contamination is still present. All the processing was the same as in the case of the first HPHT O-ND, results of which are shown in the main text. The only difference was the producer's batch. Since for the first HPHT O-ND sample the oxidation conditions were sufficient to remove nearly all the $\mathrm{sp}^{2}$-C contamination, it is probable that this sample would require longer and/or higher temperature of the air annealing to achieve a similar level of purity. $\mathrm{Sp}^{2}$ carbon is I the form of graphite with nanometric dimensions. Indeed, the spectra also show that the high-frequency "G" component is clearly composed of two lines peaking at about 1590 and $1620 \mathrm{~cm}^{\text {- }}$ ${ }^{1}$, more or less independently of the excitation wavelength. The latter one is expected as a defectinduced " $\mathrm{D}$ " mode in micro- and nanocrystalline graphite ${ }^{3}$. Strictly speaking, the " $\mathrm{D}$ " mode is dispersive. In agreement with previous works ${ }^{3}$, the " $D$ " band position was found to be strongly dispersive, peaking at about 1320, 1350 and $1405 \mathrm{~cm}^{-1}$ when the excitation wavelength was tuned from 785 to $325 \mathrm{~nm}$. Here, we emphasize that this " $D$ " band is observed at about $1320 \mathrm{~cm}^{-1}$ at $785 \mathrm{~nm}$, but must not be confused with the $T_{2 g}$ mode of the diamond. Moreover, the " $D$ " and " $G$ " bands intensity seems to increase with the decreasing particle size from c1 to uc3 fraction. 
(a)

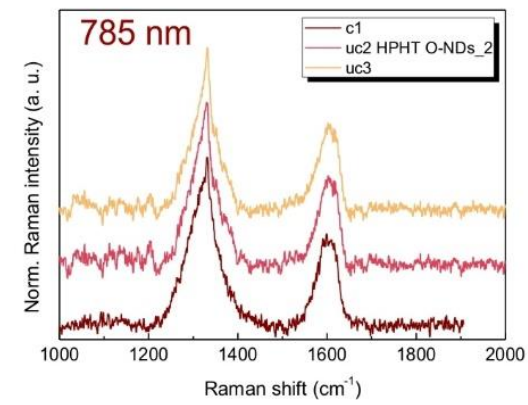

(b)

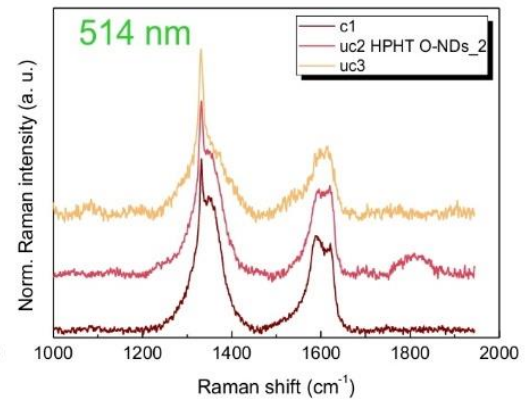

(c)

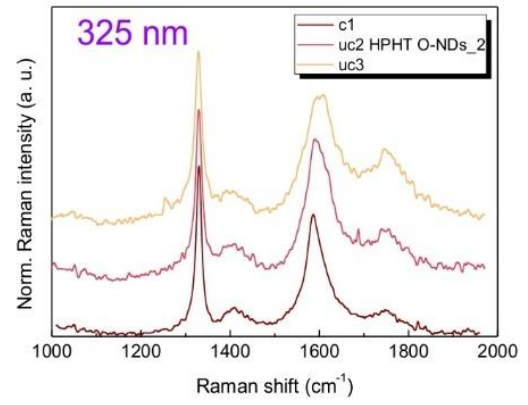

Figure S5. Multiwavelength Raman spectra of the three fraction of the second HPHT O-NDs sample set having more $s^{2}-C$. The spectra were acquired with $785 \mathrm{~nm}(a), 514 \mathrm{~nm}$ (b), and $325 \mathrm{~nm}$ (c) excitation. The spectra are shown after a baseline subtraction.

\section{Raman analysis of the SR O-DNDs fractions with $442 \mathrm{~nm}$ excitation}

Figure S6a shows the Raman spectra of the reference SD-DND sample and three fraction of the SR ODNDs acquired under $442 \mathrm{~nm}$ excitation. The spectra evidence inherent decrease of the Raman signal/PL background ratio due to the decreasing size of the $c 1$ to uc3 SR O-DNDs fractions. This effect makes any baseline subtraction and spectra evaluation very uncertain. Note also that a periodic interference pattern completely ruins the spectra after the baseline subtraction (Figure S4b).

(a)

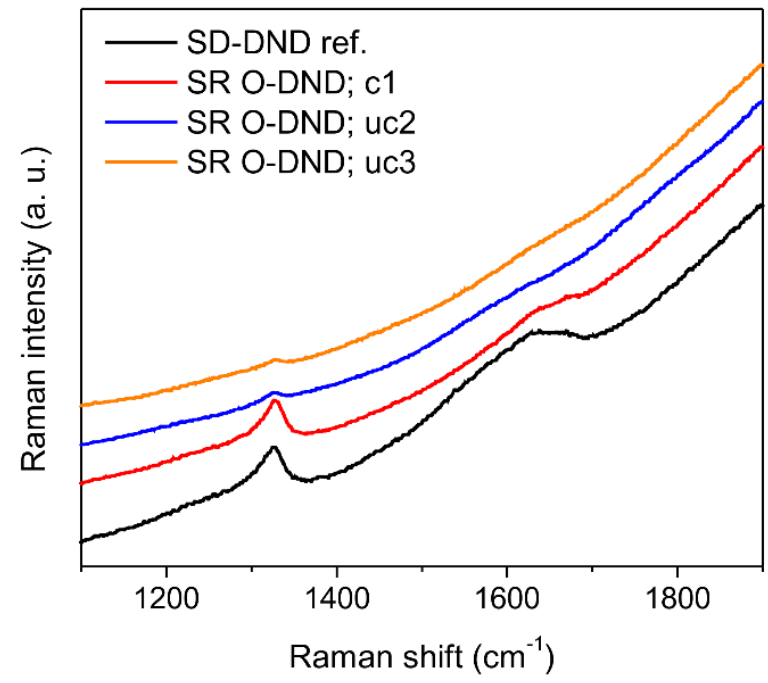

(b)

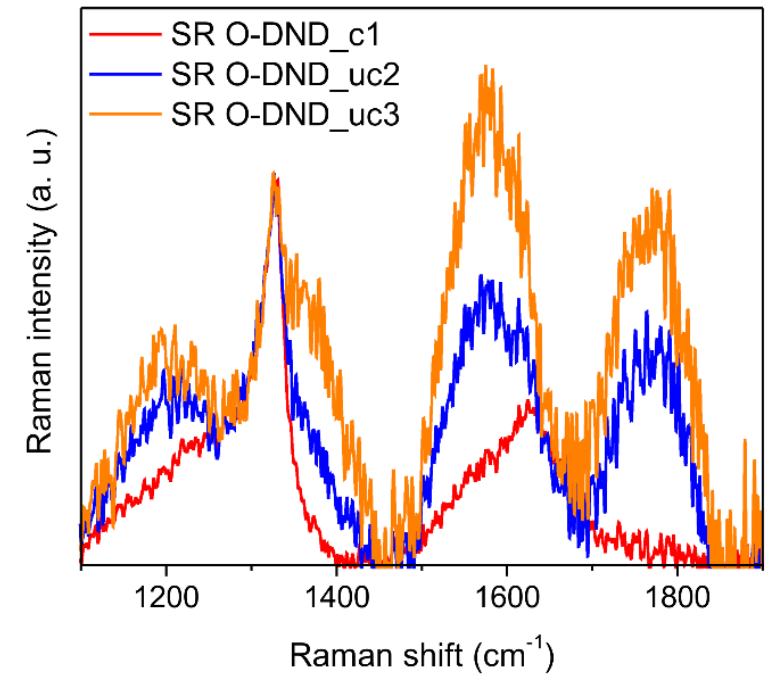

Figure S6. Raman spectra of the SD-DND reference and three fraction of the SR O-DNDs acquired with $442 \mathrm{~nm}$ excitation (a). The spectra of the SR O-DNDs C1, uc2 and uc3 fractions after the baseline subtraction.

\section{Low-frequency modes of the HPHT O-NDs fractions by $532 \mathrm{~nm}$ excitation}

Figure S7 shows raw broad-range Stokes-side Raman spectra of the HPHT O-NDs c1, uc2 and uc3 fractions obtained with $532 \mathrm{~nm}$ excitation using Bragg rejection filters. The spectra clearly document evolution of the low-frequency Raman scattering signals with the decreasing ND size. The lowfrequency region is shown in the inset in detail. In the spectrum of the $c 1$ fraction only a shoulder is apparent in the vicinity of the excitation line, while both uc2 and uc3 samples show a broad peaks with 
distinct maxima in the $50-100 \mathrm{~cm}^{-1}$ range. Moreover, the maxima shift to higher Raman shifts with decreasing size, i.e. in the expected way to be considered as confined acoustic modes of the whole particles. Please note also negligible signals of $\mathrm{sp}^{2}-\mathrm{C}$ carbon in both uc2 and uc3 samples.

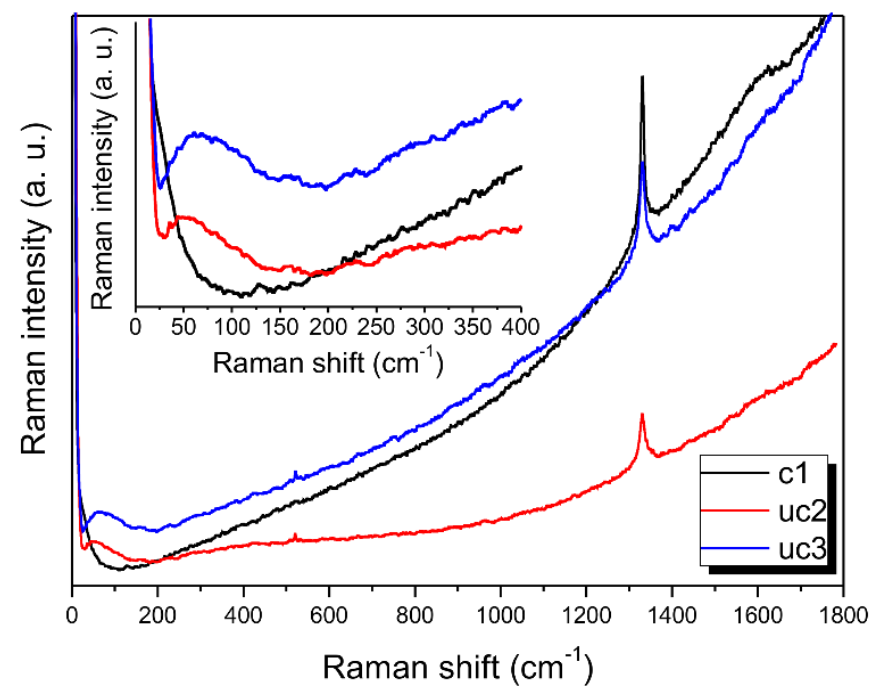

Figure S7. Broad range Raman spectra of the HPHT O-NDs $c 1$, uc2 and uc3 fractions obtained with $532 \mathrm{~nm}$ excitation using Bragg rejection filters. The inset shows the low-frequency region in detail.

\section{References}

(1) Merritt, M.; Zhang, Y. Interior-Point Gradient Method for Large-Scale Totally Nonnegative Least Squares Problems. J Optim Theory App/ 2005, 126 (1), 191-202. https://doi.org/10.1007/s10957-005-2668-z.

(2) Beaucage, G. Combined Small-Angle Scattering for Characterization of Hierarchically Structured Polymer Systems over Nano-to-Micron Meter. In Polymer Science: A Comprehensive Reference; Elsevier, 2012; pp 399-409. https://doi.org/10.1016/B978-0-444-53349-4.00032-7.

(3) Pimenta, M. A.; Dresselhaus, G.; Dresselhaus, M. S.; Cançado, L. G.; Jorio, A.; Saito, R. Studying Disorder in Graphite-Based Systems by Raman Spectroscopy. Phys. Chem. Chem. Phys. 2007, 9 (11), 1276-1290. https://doi.org/10.1039/B613962K. 